\title{
Trypanosomatid parasites rescue heme from endocytosed hemoglobin through lysosomal HRG transporters
}

María Cabello-Donayre,

Sophie Malagarie-Cazenave, ${ }^{\dagger}$

Jenny Campos-Salinas, ${ }^{\dagger}$ Francisco J. Gálvez, ${ }^{\dagger}$

Alba Rodríguez-Martínez, Estela Pineda-Molina,

Lina M. Orrego, Marta Martínez-García,

María P. Sánchez-Cañete, Antonio M. Estévez and

José M. Pérez-Victoria*

Instituto de Parasitología y Biomedicina "López-

Neyra”, CSIC, (IPBLN-CSIC), PTS Granada, Avda.

del Conocimiento s/n, Granada 18016, Spain.

\section{Summary}

Pathogenic trypanosomatid parasites are auxotrophic for heme and they must scavenge it from their human host. Trypanosoma brucei (responsible for sleeping sickness) and Leishmania (leishmaniasis) can fulfill heme requirement by receptor-mediated endocytosis of host hemoglobin. However, the mechanism used to transfer hemoglobin-derived heme from the lysosome to the cytosol remains unknown. Here we provide strong evidence that HRG transporters mediate this essential step. In bloodstream T. brucei, TbHRG localizes to the endolysosomal compartment where endocytosed hemoglobin is known to be trafficked. TbHRG overexpression increases cytosolic heme levels whereas its downregulation is lethal for the parasites unless they express the Leishmania orthologue LmHR1. LmHR1, known to be an essential plasma membrane protein responsible for the uptake of free heme in Leishmania, is also present in its acidic compartments which colocalize with endocytosed hemoglobin. Moreover, LmHR1 levels modulated by its overexpression or the abrogation of an LmHR1 allele correlate with the mitochondrial bioavailability of heme from lysosomal hemoglobin. In addition, using heme auxotrophic yeasts we show that TbHRG and LmHR1 transport hemoglobin-derived heme from the digestive vacuole to the cytosol. Collectively, these results show that

Accepted 29 May, 2016. *For correspondence. E-mail josepv@ipb. csic.es; Tel. +34 958181658; Fax +34 958181632. ${ }^{\dagger}$ These authors contributed equally to this work. trypanosomatid parasites rescue heme from endocytosed hemoglobin through endolysosomal HRG transporters, which could constitute novel drug targets.

\section{Introduction}

Protozoa from the trypanosomatid family includes parasites responsible for dramatic neglected diseases in humans and important illnesses in animals. The most important members of this family are Leishmania spp., which cause different forms of leishmaniasis in humans and dogs, Trypanosoma brucei, responsible for sleeping sickness in humans and nagana in cattle, and Trypanosoma cruzi, the etiological agent of Chagas disease in humans. These parasites have digenetic life cycles, alternating between two hosts. In the mammalian host, amastigote forms of Leishmania spp. and T. cruzi are intracellular, whereas bloodstream forms of $T$. brucei are extracellular. In the insect vector, all forms (Leishmania promastigotes, T. cruzi epimastigotes and T. brucei procyclic forms) are extracellular. Chemotherapy remains the main weapon to fight these diseases, but today's arsenal has many limitations as current drugs are generally toxic and hampered by the increasing problem of drug resistance. Therefore, there is an urgent need to develop new medicines in the fight against these neglected diseases.

A rational approach to discovering new drug targets effective against trypanosomatid parasites consists of exploiting their inability to synthesize the essential compound heme, which they have to salvage from their hosts. This absolute dependence on heme rescue was indeed considered their Achilles' heel more than 80 years ago (Lwoff, 1933). In recent years, some light has been shed on the parasites' heme uptake mechanism. Bloodstream forms of $T$. brucei do not internalize free heme (Vanhollebeke et al., 2008). In contrast, they acquire heme from hemoglobin $(\mathrm{Hb})$, which is taken up through the receptor ( $\mathrm{TbH} \mathrm{pHbR}$ )-mediated endocytosis of haptoglobin ( $\mathrm{Hp})-\mathrm{Hb}$ complexes (Vanhollebeke et al., 2008). This complex follows the endocytic route to late 
endosomes and lysosome, where $\mathrm{Hb}$ is digested. The heme moiety released is then exported by an unknown mechanism to the cytosol where it is incorporated into the parasite's hemeproteins, such as sterol $14 \alpha$ demethylase (Lepesheva et al., 2010). Interestingly, although this mechanism facilitates heme rescue from the host, it is also responsible for the human's innate immunity to most African trypanosomes (Vanhollebeke et al., 2008; Stodkilde et al., 2014). This is because $\mathrm{TbHpHbR}$ also recognizes the complex between $\mathrm{Hb}$ and $\mathrm{Hp}$-related protein $(\mathrm{Hpr})$, which is present in human HDL particles that also contain the trypanolytic factor apoL1. Only two $T$. brucei subspecies $(T$. b. rhodesiense and $T$. b. gambiense) can evade this defense mechanism and ultimately cause sleeping sickness in humans (Pays et al., 2014). In contrast, procyclic forms of T. brucei, which reside in the tsetse fly, are able to accumulate free heme but cannot internalize $\mathrm{Hp}-\mathrm{Hb}$ as they do not express $\mathrm{TbHpHbR}$ (Vanhollebeke et al., 2008). In the case of $T$. cruzi epimastigotes, heme and $\mathrm{Hb}$ internalization take place via different routes (Lara et al., 2007). Heme uptake proceeds faster than $\mathrm{Hb}$ internalization (Lara et al., 2007) and is affected by ABC transporter inhibitors such as cyclosporine A (Lara et al., 2007; Cupello et al., 2011), whereas $\mathrm{Hb}$ is internalized through the endocytic pathway (Lara et al., 2007). Finally, promastigotes (Chang and Chang, 1985) and axenic amastigotes (Carvalho et al., 2009) of Leishmania also take heme from two sources, heme and $\mathrm{Hb}$, which can be also used as a source of iron (Carvalho et al., 2009). These compounds are internalized through two independent routes, the faster mechanism is the one involving free heme (Campos-Salinas et al., 2011). Hb is captured by receptor-mediated endocytosis (Sengupta et al., 1999; Krishnamurthy et al., 2005) and trafficked to the lysosome in a process mediated by Rab5 (Singh et al., 2003) and Rab7 (Patel et al., 2008). Heme is then released from digested $\mathrm{Hb}$ (Patel et al., 2008), it exits the lysosome by an unknown mechanism and reaches the mitochondria, with the $A B C$ transporter LABCG5 playing an essential role in this trafficking activity (Campos-Salinas et al., 2011). This pathway is an attractive therapeutic target: not only Leishmania requires degradation of endocytosed $\mathrm{Hb}$ for their growth (Patel et al., 2008; Campos-Salinas et al., 2011; Guha et al., 2013), but also the $\mathrm{Hb}$ receptor-encoding DNA is a promising vaccine candidate for human use to prevent leishmaniasis (Guha et al., 2013). The essential mitochondrial $A B C$ transporter $L m A B C B 3$, responsible for cytosolic iron/sulfur clusters biogenesis, also plays a role in mitochondrial heme metabolism (Martinez-Garcia et al., 2016). Regarding free hemin, it is imported by the heme transporter LHR1, described in L. amazonensis (Huynh et al., 2012). The heterologous expression of
LHR1 in a Saccharomyces cerevisiae strain auxotrophic for heme facilitates yeast growth in the presence of low extracellular hemin concentrations by promoting porphyrin uptake (Huynh et al., 2012). LHR1 localizes at the plasma membrane, as expected for a porphyrin importer, but also at the lysosomal compartments of promastigote and amastigote forms of Leishmania. This dual localization is also observed in mammalian cells when LHR1 is expressed heterologously (Huynh et al., 2012). Whether lysosomal LHR1 is physiologically relevant or is a product of its forced overexpression remains to be seen. Besides, LHR1 is essential for the parasite, and even the ablation of one allele strongly prevents the virulence of the parasite in animal models of cutaneous leishmaniasis, underlining its potential as a new drug target (Huynh et al., 2012; Miguel et al., 2013; Renberg et al., 2015).

LHR1 belongs to the HRG (Heme Response Genes) family of heme transporters, first described in Caenorhabditis elegans (Rajagopal et al., 2008). In this worm, CeHRG4 is a heme importer at the plasma membrane whereas intracellular CeHRG1 could exports heme from the lysosome to the cytosol (Rajagopal et al., 2008; Yuan et al., 2012). Similarly, macrophage HRG1 mediates heme export from the phagolysosome into the cytosol during erythrophagocytosis of senescent red cells in mammals (Delaby et al., 2012; White et al., 2013). All these HRG proteins show low similarity with LHR1 (around 14\% identity). LHR1 syntenic genes are also present in both species of Trypanosoma (Huynh et al., 2012) and so a role in heme trafficking could be expected.

In this work, we have characterized the TbHRG protein of bloodstream forms of $T$. brucei and analyzed the functional role of lysosomal LHR1 from Leishmania. Functional assays in homologous and heterologous systems strongly suggest that both TbHRG and LHR1 are the lysosomal transporters responsible for heme salvage from endocytosed $\mathrm{Hb}$. As these proteins are essential in T. brucei and Leishmania, and as they present a very low degree of similarity with human HRG1, their specific targeting could represent a novel approach to controlling the neglected diseases derived from these parasites.

\section{Results}

\section{Bloodstream forms of T. brucei express an essential HRG protein in the endolysosomal compartment}

The genome of $T$. brucei presents a syntenic gene of the Leishmania heme transporter gene LHR1, (Tb927.8.6010) which we have called TbHRG. Its protein product TbHRG is $100 \%$ identical in the cattle pathogen $T$. brucei brucei and in the human pathogen $T$. brucei gambiense, and only shows a moderate 
degree of similarity with other HRG proteins outside the Trypanosoma genus (Fig. $1 \mathrm{~A}$ and SI Appendix Table S1): around $22 \%$ identity with Leishmania HR1, 13\% identity with $C$. elegans HRG4, 9\% identity with C. elegans HRG1 and $16 \%$ identity with human HRG1. The related parasite $T$. cruzi also has a LHR1 syntenic gene (with only six amino acid substitutions in the two diploid alleles, TcHRGa and TcHRGb, of the hybrid strain sequenced), and show $32 \%$ identity with the T. brucei transporter. TbHRG is a $18.1 \mathrm{kDa}$ molecular weight protein and, like other HRG proteins, it is expected to contain four transmembrane domains (Fig. 1A).

We observed, using real-time quantitative PCR (RTqPCR), that TbHRG was constitutively expressed in both stages of $T$. brucei, the expression in the parasite's insect stage being eightfold higher than in bloodstream forms (7.9 \pm 1.3 fold, $P<0.05$ ). The expression of $T b H R G$ in the clinically relevant bloodstream form of the parasite was striking, as T. brucei is unable to take up free heme in this stage (Vanhollebeke et al., 2008). Therefore, the physiological function of TbHRG could differ from the one proposed for LHR1, which imports heme from the environment (Huynh et al., 2012). Thus, we analyzed the importance of TbHRG function in bloodstream-form parasites by inducible gene knockdown via RNA interference (RNAi). After adding the inducer doxycycline, the decreased expression of the gene $(66 \%, P<0.005)$ correlated with an arrest in the parasite's growth (Fig. 1B, left). This growth defect was accompanied by significant phenotypic alterations in the morphology of the induced parasites, as shown in Fig. 1B (right), strongly suggesting that TbHRG is essential in bloodstream-form T. brucei, and it is in agreement with high-throughput RIT-seq data (Alsford et al., 2011).

We then analyzed the cellular distribution of TbHRG in T. brucei. To that end, we overexpressed a C-terminal GFP-fused version of the protein and analyzed its localization in the parasite using fluorescence microscopy. Figure $1 \mathrm{C}$ shows that TbHRG was not observed at the plasma membrane but was actually located intracellularly. The GFP-tagged protein colocalized with Dextran Alexa 647, a fluid-phase endocytosis marker, indicating that it was located in the parasite's endolysosomal compartment. As endocytosed $\mathrm{Hb}-\mathrm{Hp}$ complexes are trafficked to the parasite late endosome-lysosome (Widener et al., 2007), the essential role of TbHRG could be related to the export of $\mathrm{Hb}$-derived heme to the cytosol.

\section{TbHRG is a heme transporter}

To study whether or not TbHRG transports heme, we analyzed the ability of TbHRG to rescue the growth defect of a heme auxotrophic $S$. cerevisiae line. This yeast strain has a deletion in hem1, the first gene of the heme biosynthetic pathway (Kohut et al., 2011). As S. cerevisiae cannot internalize heme efficiently from the environment, hem1 $1 \Delta$ yeast must grow in the presence of either ALA, the product of the deleted hem1 enzyme, or high quantities of hemin, which can enter the cells when at higher concentrations via an unknown mechanism.

We first expressed a GFP-tagged version of TbHRG in a hem1 $1 \Delta$ yeast strain grown in the presence of ALA. TbHRG-GFP was distributed in both the yeast plasma membrane and in its digestive vacuole, which is equivalent to the trypanosomatid lysosome (Fig. 2A). Control and TbHRG-expressing hem1 $1 \Delta$ yeasts were then plated in an ALA-free medium with increasing concentrations of hemin. Figure 2B (upper panel) shows that TbHRG was able to rescue the growth of the mutant yeast at low hemin concentrations $(0.25 \mu \mathrm{M})$, as described for LaHR1 (Miguel et al., 2013) and other HRG proteins (Yuan et al., 2012), strongly suggesting that TbHRG is indeed a heme transporter.

\section{TbHRG transports the heme released after $\mathrm{Hb}$ breakdown to the yeast cytoplasm}

In yeast, endocytosed $\mathrm{Hb}$ is trafficked to the vacuole, where it is digested (Weissman et al., 2008). Subsequently the released heme is not efficiently transported to the cytosol but rather it is degraded in the lumen of the vacuole (Weissman et al., 2008). To assess the possibility that lysosomal/ vacuolar TbHRG transports heme produced by the digestion of $\mathrm{Hb}$ from these organelles to the cytosol, we evaluated the ability of TbHRG to rescue the growth of hem1 $1 \Delta$ yeast in the presence of $\mathrm{Hb}$. Hem $1 \Delta$ control yeast cells did not grow in the presence of $0.025 \mu \mathrm{M} \mathrm{Hb}$ (equivalent to 0.1 $\mu \mathrm{M}$ heme), and require higher $\mathrm{Hb}$ concentrations before initiating growth (Fig. 2B, lower panel). Contrastingly, mutant yeast cells expressing TbHRG were able to grow at this low $\mathrm{Hb}$ concentration and showed a significant improvement in growth at higher $\mathrm{Hb}$ concentrations (Fig. 2B, lower panel). Importantly, TbHRG did not increase $\mathrm{Hb}$ uptake in the mutant yeast (data not shown). Finally, we also measured TbHRG-mediated bioavailability of $\mathrm{Hb}$-derived heme by measuring the activity of a heme-dependent enzyme, such as catalase. As yeast catalases are hemeproteins regulated by heme and they are not present into the digestive vacuole, catalase activity can be used as an endogenous marker for measuring intracellular heme levels (Kathiresan et al., 2014). We measured yeast catalase activity with the simple assay developed for intact bacterial isolates and human cells (Iwase et al., 2013), consistent in the visualization of the foam produced by the oxygen bubbles generated from the decomposition of hydrogen peroxide by catalase, trapped by a detergent. The foam's height in the test-tubes 

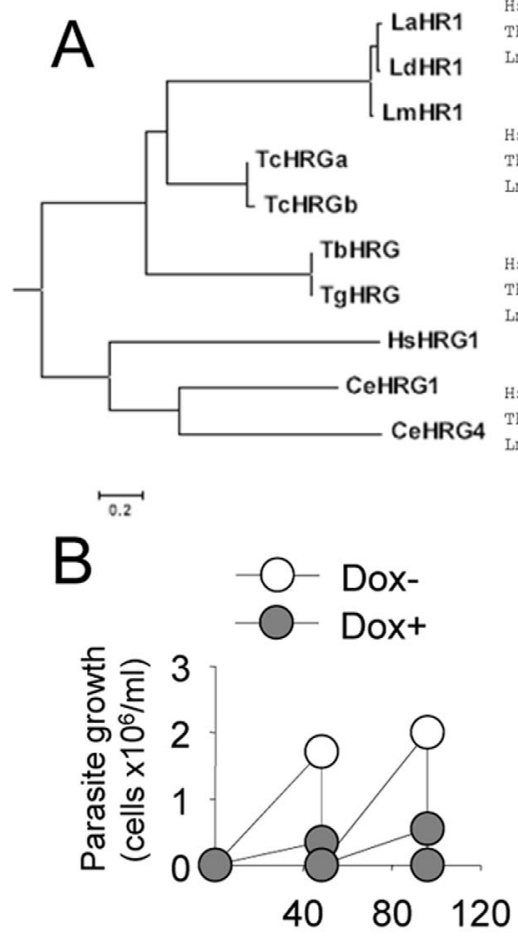

Time after induction (hours)
HSHRG1 MAPSRLQLGLRAAYSGISSVAGESIFLVWTVVIRQ

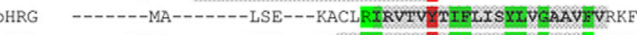
LMHR1 -----MNDL-------ERK---RRTFRIWLAI IIIFALAYISGVLIEFCFVH

HSHRG1 PGTA MG TLLAGVLALWVEVTHVMYMQDY--WRT-WLKGLRGFFFVGVLFA

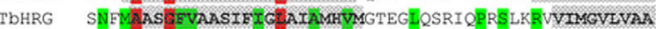
LMHR1 ONYWW CGEGFVVIWLIA

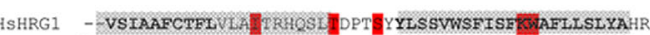

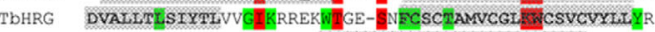

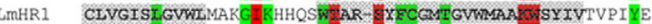

ASHRG1 YRADEADI---SILSDF---------------------

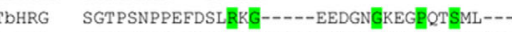

MHR1 LREDTLEE-DALIBLGIIDSEKNEASGNNERVISYMENCA

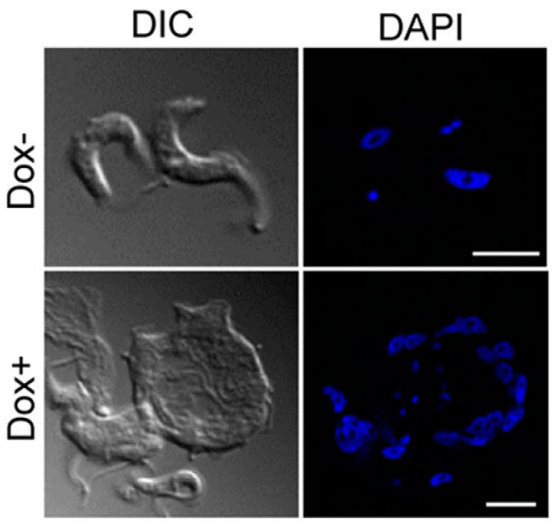

C

TbHRG-GFP

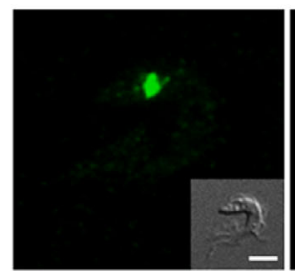

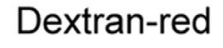

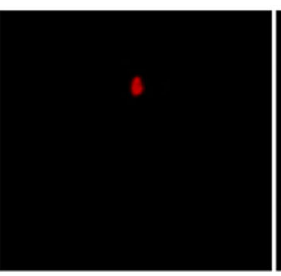

Merge

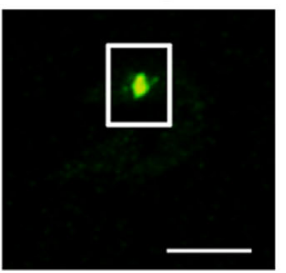

Coloc.

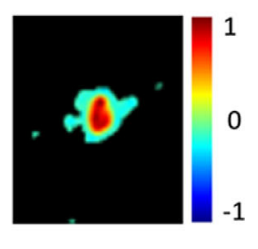

Fig. 1. Bloodstream T. brucei parasites express an essential HRG protein located in the endololysosomal compartment of the parasite. A. The genome of $T$. brucei contains a HRG gene that shows a low similarity with HRG genes from other organisms. Left. Phylogenetic analysis of HRG sequences from L. amazonensis [LaHR1, (Huynh et al., 2012)], L. donovani (LdHR1, accession number XP_003861320. 1), L. major (LmHR1, accession number XP_001683741.1), T. brucei brucei (TbHRG, accession number XP_847431.1), T. brucei gambiense (TgHRG, accession number XP_011775941.1), T. cruzi (TcHRGa, accession number XP_814012.1, and TcHRGb, accession number XP_804283.1), H. sapiens (HsHRG1, accession number NP_060312.2) and C. elegans (CeHRG1, accession number NP_508690.2 and Ce-HRG4, accession number NP_502055.1). Aligned protein sequences were subjected to phylogenetic analysis using the Maximum Likelihood method of the MEGA 6 software. Right. Amino acids sequence alignment for HsHRG1, TbHRG and LmLHR1 (ClustalW software) highlighting the identical aminoacid shared in these three transporters (red) and in HRG from parasites (green). The transmembrane domains found in all transporters are highlighted in grey.

B. TbHRG is essential in bloodstream T. brucei. Left. Growth curves of TbHRG-depleted bloodstream trypanosomes after RNAi induction with

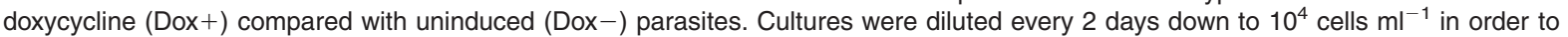
maintain the cells exponential growth. Right. Cell morphology effect in bloodstream trypanosomes upon TbHRG knockdown visualized by DIC optic and fluorescence microscopy of DAPI-stained parasites $48 \mathrm{~h}$ after RNAi induction with doxycicline (Dox + ). The figure shows parasites representative of a total population with a similar morphology. Scale bar: $5 \mu \mathrm{m}$.

C. TbHRG is located in the endolysosomal compartment of the parasite. T. brucei cells expressing TbHRG-GFP (green) were incubated at $28^{\circ} \mathrm{C}$ with the endocytic marker Dextran-Alexa 647 (Dextran-red) for $30 \mathrm{~min}$ and then their colocalization (yellow) was analyzed (merge). The figure shows a parasite representative of a total population of parasites with a similar fluorescence pattern. Nomarsky images are shown in the inset. Scale bar: $5 \mu \mathrm{m}$. The colocalization color map (right) shows the intensity of colocalization between TbHRG-GFP and the redlabelled dextran marker as indicated by the color bar. In these colour maps, the -1 to +1 heat map depicts the measured Icorr values. Negatively correlated relationships (Icorr values between -1 and 0 ) are shown in blue-green colors, whereas positive correlations (Icorr values between 0 and 1) are represented by warmer yellow-red colors. 
A

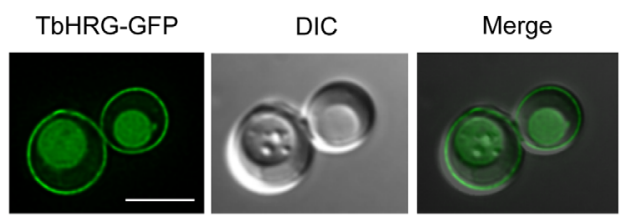

B

$\mu \mathrm{M}$ Heme

\begin{tabular}{|c|c|c|c|c|c|}
\hline & 0 & 0.1 (as Hemin) & 0.25 (as Hemin) & 1 (as Hemin) & ALA \\
\hline Control & & & 0 & $0 \Omega$ & 00 눙 \\
\hline TbHRG & & & $0 \cdot \%$ & $00 \%$ & $00 \%$ \\
\hline & 0 & 0.1 (as $\mathrm{Hb}$ ) & 0.25 (as $\mathrm{Hb}$ ) & 1 (as Hb) & ALA \\
\hline Control & & & 00 & 00 & $000 \%$ \\
\hline TbHRG & & 00 & 00 & 우 0 站 & 1006 \\
\hline
\end{tabular}

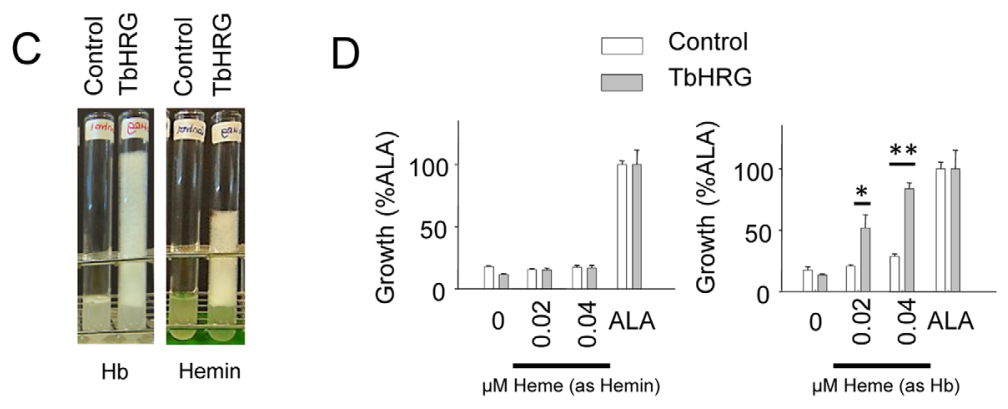

Fig. 2. TbHRG mediates Hb-derived heme transport from the digestive vacuole of yeast to the cytosol.

A. TbHRG heterologously expressed in a heme auxotrophic $S$. cerevisiae strain (hem1 1 ) shows a double localization at the plasma membrane and the digestive vacuole. The figure shows a yeast cell expressing TbHRG-GFP (green) representative of a total population of cells with a similar fluorescence pattern. Nomarsky images are shown in the central panel. Scale bar: $5 \mu \mathrm{m}$.

B. TbHRG enables hem1 $1 \Delta$ yeast to use endocytosed $\mathrm{Hb}$ as a source of heme. Hem1 $1 \Delta$ yeast transformed with empty plasmid (control) or plasmid containing TbHRG were spotted in 10-fold serial dilutions on plates supplemented with different concentrations of hemin (up) or $\mathrm{Hb}$ (down). Positive control: $250 \mu \mathrm{M}$ ALA.

C. TbHRG increased yeast cytosolic heme levels after $\mathrm{Hb}$ endocytosis. Catalase activity, observed as foam formation as described in Materials and Methods, of hem $1 \Delta$ yeast transformed with empty (control) or TbHRG containing plasmid (TbHRG) and incubated with $0.5 \mu \mathrm{M}$ of $\mathrm{Hb}$ (left) or $2 \mu \mathrm{M}$ hemin (right). Picture representative of three independent experiments.

D. TbHRG-expressing yeast obtained heme more efficiently from $\mathrm{Hb}$ than from hemin. Hem1 $1 \Delta$ yeast transformed with the same plasmids and incubated for $24 \mathrm{~h}$ in a liquid medium with the indicated concentrations of hemin (left) or $\mathrm{Hb}$ (right). Positive control: $250 \mu \mathrm{M}$ ALA. The results represent the mean \pm SD of three independent experiments with $P$ values ${ }^{*}<0.05$ and ${ }^{* *}<0.005$.

correlates well with the sample's catalase activity (Iwase et al., 2013). Figure $2 \mathrm{C}$ shows that hem $1 \Delta$ control yeast incubated in the presence of $0.5 \mu \mathrm{M} \mathrm{Hb}$ had a very low catalase activity, even though the given $\mathrm{Hb}$ concentration allowed cell growth of the mutant yeast. By contrast, hem1s yeast cells expressing TbHRG and incubated under the same conditions produced a significant increase in catalase activity, as shown by the foam developed in the tube (Fig. 2C). These results strongly suggest that vacuolar TbHRG was involved in salvaging heme from internalized $\mathrm{Hb}$. Hem1s yeast incubation with an equivalent hemin concentration also only resulted in catalase activity when TbHRG was expressed, although at lower levels than those obtained with $\mathrm{Hb}$ (Fig. 2C). This supports the idea that plasma membrane TbHRG was also functional.
This catalase assay suggested that $\mathrm{Hb}$-derived heme was used more efficiently by TbHRG-expressing yeast than hemin. To confirm this point, control and TbHRGexpressing hem1s yeast cells were incubated in a liquid medium containing a low supplement of heme, either as hemin or as $\mathrm{Hb}$, and then their degrees of growth compared. The hem1s strain, whether expressing TbHRG or not, did not grow at the low hemin concentrations assayed (0.02 and $0.04 \mu \mathrm{M})$ (Fig. 2D, left). On the other hand, supplementation with $\mathrm{Hb}$ significantly improved the growth of the TbHRG-expressing strain in the presence of only $0.02 \mu \mathrm{M}$ heme $(0.005 \mu \mathrm{M} \mathrm{Hb})$, and completely restored growth levels at $0.04 \mu \mathrm{M}$ heme $(0.01$ $\mu \mathrm{M} \mathrm{Hb}$ ) (Fig. 2D, right). These results confirm that yeast cells expressing TbHRG were able to obtain heme more 


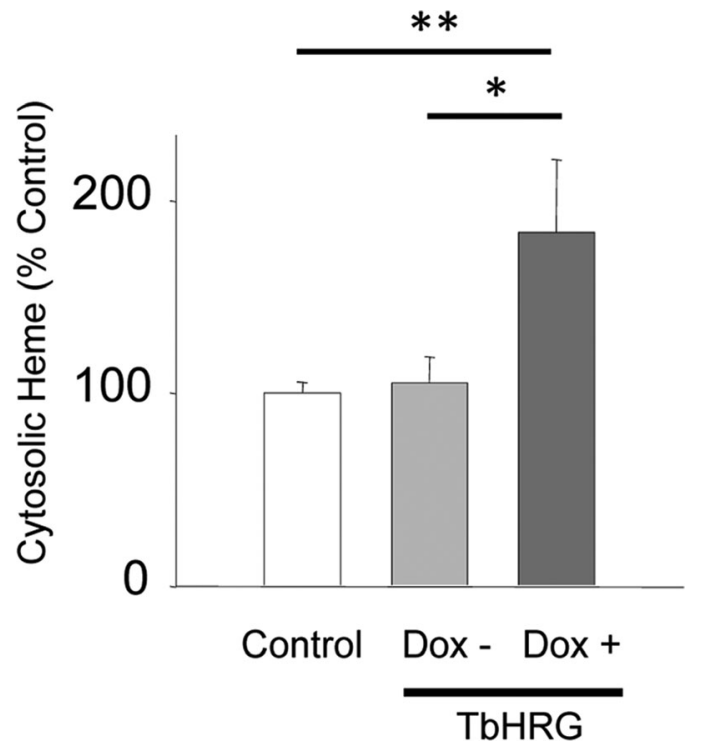

Fig. 3. Lysosomal TbHRG increases cytosolic $\mathrm{Hb}$-derived heme levels in T. brucei. Cytosolic heme levels were measured as described in Materials and Methods after incubating the indicated T. brucei strains in culture medium including $10 \%$ hiFBS as a source of $\mathrm{Hp}-\mathrm{Hb}$. TbHRG-GFP expression was induced (Dox + ) or not induced (Dox-) with doxycycline. The results represent the mean \pm SD of three independent experiments. $P$ values ${ }^{*}<0.05$ and ${ }^{* *}<0.005$.

efficiently from $\mathrm{Hb}$ through vacuolar TbHRG than from hemin through plasma membrane TbHRG.

Lysosomal TbHRG increase cytosolic heme levels from endocytosed $\mathrm{Hb}$ in $\mathrm{T}$. brucei

Altogether, these results strongly suggested that endolysosomal TbHRG transport Hb-derived heme to the cytosol of bloodstream forms of $T$. brucei. Therefore, we expected that TbHRG overexpressing parasites grew in the presence of $\mathrm{Hp}-\mathrm{Hb}$ containing serum contain a higher heme concentration in their cytosol. To check it, we measured heme levels in the cytosol after gentle lysis of the parasites overexpressing of not TbHRG, as described in (da Silva Augusto et al., 2015). Figure 3 shows that TbHRG overexpressing parasites (after induction with doxicyclin) contained around $85 \%$ more cytosolic heme levels than Wt or uninduced parasites $(P<0.005)$.

\section{Leishmania LmHR1 complements TbHRG deficiency in bloodstream-form T. brucei}

In Leishmania, LHR1 is located at the plasma membrane and imports heme from the environment (Huynh et al., 2012). Although this function differs from the one proposed here for TbHRG, LHR1 is also located at the parasite's lysosome (Huynh et al., 2012) and a similar role in the rescue of heme from endocytosed $\mathrm{Hb}$ could be expected. To investigate this point, we first analyzed the ability of $L$. major HR1 (LmHR1) to rescue the essential role of endolysosomal TbHRG in T. brucei. Firstly, a Cterminal GFP-tagged version of LmHR1 was cloned in a T. brucei expression plasmid and transfected in the TbHRG RNAi T. brucei clone described above. Unlike LaHR1 localization in L. amazonensis (Huynh et al., 2012), LmHR1-GFP was not found at the plasma membrane of bloodstream-form T. brucei (Fig. 4A). As with TbHRG-GFP, LmHR1-GFP showed an intracellular location corresponding to the endolysosome compartment, as deduced from its colocalization with fluorescent Dextran (Fig. 4A). Furthermore, the lethal effect produced by RNAi-mediated ablation of TbHRG after the addition of doxycycline was mostly circumvented by the expression of LmHR1 (Fig. 4B), indicating that LmHR1 can complement TbHRG function at the endolysosomal compartment of $T$. brucei. RT-qPCR confirmed TbHRG underexpression $(52 \%, P<0.03)$ after the addition of doxycycline.

Vacuolar LmHR1 also rescues heme from endocytosed $H b$ in yeast

To further confirm that LHR1 could have a similar function to TbHRG, we analyzed its ability to rescue the
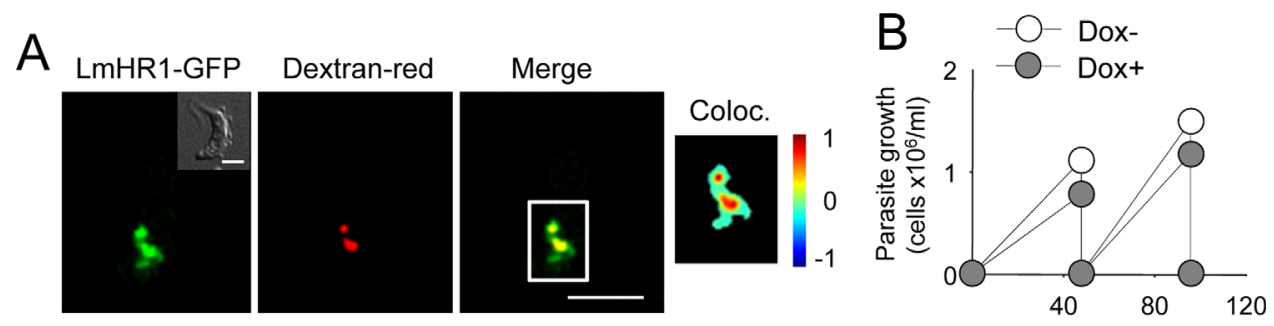

Time after induction (hours)

Fig. 4. LmHR1 complements TbHRG function in T. brucei.

A. Heterologously expressed LmHR1-GFP is located in the endolysosomal compartment of bloodstream T. brucei. Bloodstream T. brucei expressing LmHR1-GFP (green) were incubated at $37^{\circ} \mathrm{C}$ with Dextran-Alexa 647 (Dextran-red) for 30 min and observed using fluorescence microscopy as indicated in Fig. $1 \mathrm{C}$.

B. LmHR1 complements TbHRG function in T. brucei. Growth curves of LmHR1-GFP expressing and TbHRG-depleted bloodstream trypanosomes after RNAi induction with doxycycline (Dox+) compared with uninduced (Dox-) parasites, as described in Fig. 1B. 


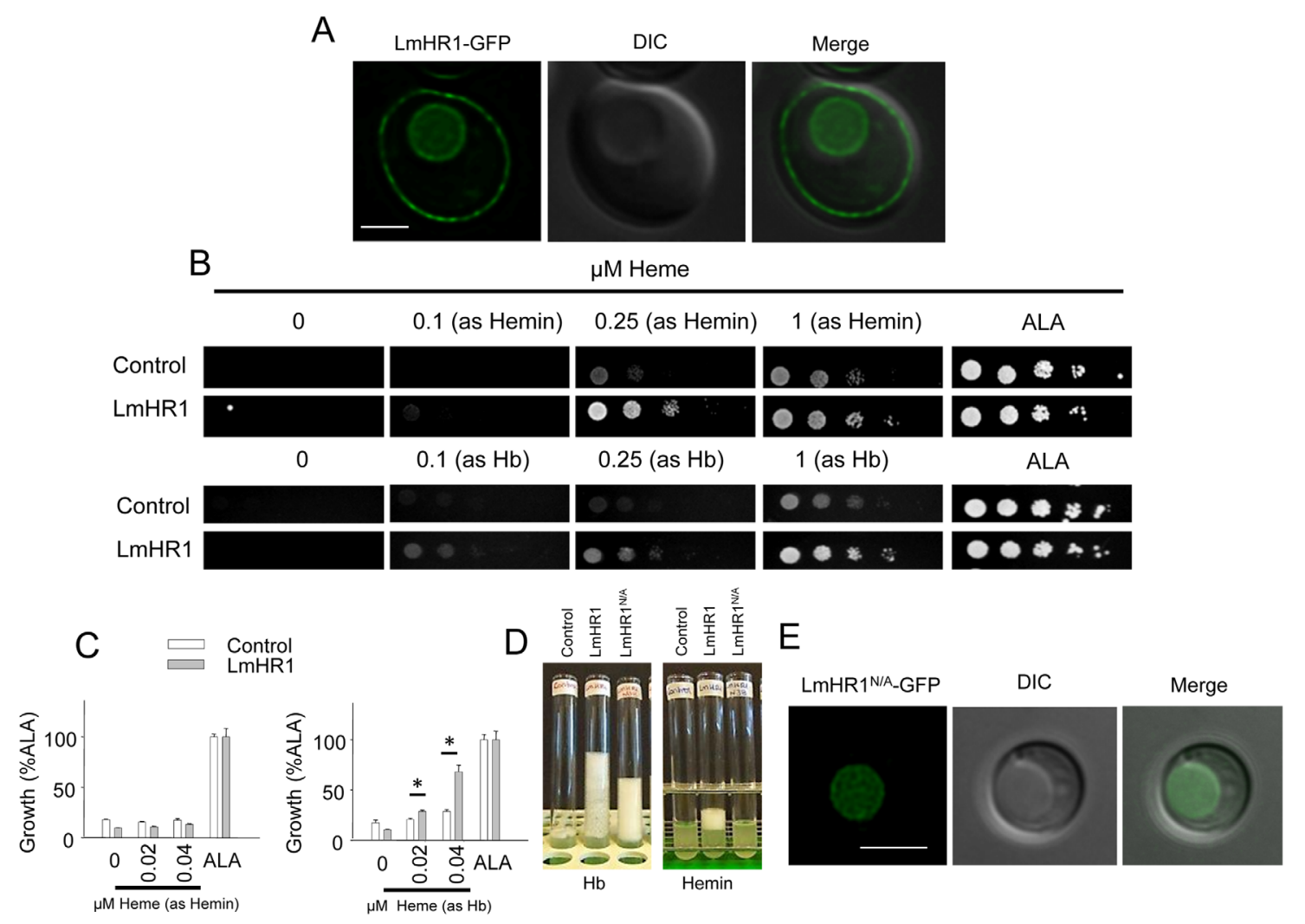

Fig. 5. LmHR1 mediates Hb-derived heme transport from the digestive vacuole of yeast to the cytosol.

A. LmHR1 heterologously expressed in hem1 $1 \Delta$ yeast shows a double localization at the plasma membrane and the digestive vacuole. LmHR1-GFP is shown in green. Nomarsky images are shown in the central panel. The figure shows a yeast cell representative of a total population of yeast with a similar fluorescence pattern. Scale bar: $5 \mu \mathrm{m}$.

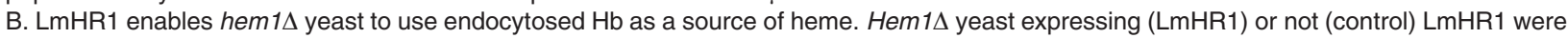
spotted in 10-fold serial dilutions on plates supplemented with different concentrations of hemin (up) or $\mathrm{Hb}$ (down) as described in Fig. 2B.

C. LmHR1-expressing yeast obtained heme more efficiently from $\mathrm{Hb}$ than from hemin. Hem1 $1 \Delta$ yeast transformed with the same plasmids and incubated for $24 \mathrm{~h}$ in a liquid medium with the indicated concentrations of hemin (left) or $\mathrm{Hb}$ (right). Positive control: $250 \mu \mathrm{M} \mathrm{ALA}$. The results represent the mean \pm SD of three independent experiments, with $P$ value ${ }^{*}<0.05$.

(D) LmHR1 mediates Hb-derived heme transport from the yeast digestive vacuole to the cytosol. Catalase activity, measured as described in Fig. $2 \mathrm{D}$, of hem $1 \Delta$ yeast expressing LmHR1 or mutant $\mathrm{LmHR} 1^{\mathrm{N} / \mathrm{A}}$ or transformed with empty plasmid (control) and incubated with $0.5 \mu \mathrm{M}$ of $\mathrm{Hb}$ (left) or $2 \mu \mathrm{M}$ hemin (right). Representative picture of three independent experiments.

(E) Mutant LmHR1 $1^{\mathrm{N} / \mathrm{A}}$ is located in the digestive vacuole of hem $1 \Delta$ yeast. LmHR1 ${ }^{\mathrm{N} / \mathrm{A}}$-GFP is shown in green and Nomarsky images are shown in the central panel. The figure shows a yeast cell representative of a total population of yeast with a similar fluorescence pattern. Scale bar: $5 \mu \mathrm{m}$.

growth of hem1 $1 \Delta$ yeast in the presence of $\mathrm{Hb}$. We first analyzed LmHR1-GFP localization in this yeast strain. Fluorescence microscopy showed a double localization at both the plasma membrane and the vacuole (Fig. $5 A$ ), as observed for TbHRG-GFP. Then we incubated control and LmHR1-expressing hem1 $1 \Delta$ yeasts in the presence of increasing concentrations of hemin and $\mathrm{Hb}$. The spot growth assay (Fig. 5B) showed that LmHR1 was able to rescue the growth of mutant yeast in the presence of both sources of porphyrin. Heme derived from $\mathrm{Hb}$ was again more efficient than hemin, as 0.01 $\mu \mathrm{M} \mathrm{Hb}$ (equivalent to $0.04 \mu \mathrm{M}$ heme) allowed $70 \%$ of the control (ALA) growth in liquid medium, whereas 0.04 $\mu \mathrm{M}$ hemin had no effect in this LmHR1-expressing hem1 $1 \Delta$ strain (Fig. 5C). As observed for TbHRG, LmHR1 expression did not increase $\mathrm{Hb}$ uptake in the mutant yeast (data not shown). Finally, the LmHR1 role in the rescue of $\mathrm{Hb}$-derived heme was measured following the catalase assay described above. Results shown in Fig. 5D indicate that LmHR1 expression significantly increased the catalase activity of yeast incubated in the presence of $0.5 \mu \mathrm{M} \mathrm{Hb}$. As observed for TbHRG, catalase activity of LmHRG-expressing yeast was higher when the source of heme was $\mathrm{Hb}$ rather than free hemin (Fig. 5D).

During the initial cloning of $L m H R 1$ in yeast expressing plasmids, one clone showed point mutations, probably caused by PCR amplification, that changed amino acid N38, located between TM1 and TM2, to Ala $\left(\mathrm{LmHR} 1^{\mathrm{N} / \mathrm{A}}\right)$. This residue was not included in the extensive mutagenesis analysis performed in LaHR1 (Renberg et al., 2015), and therefore its role was unknown. 
Although this mutant was originally discounted, we observed that the protein was well expressed but localized exclusively to the digestive vacuole (Fig. $5 \mathrm{E}$ ). If this mutation did not affect the functionality of LmHR1, it could be useful in terms of definitively assigning its role at the vacuole. Indeed, when incubated in the presence of $0.5 \mu \mathrm{M} \mathrm{Hb}, \mathrm{LmHR} 1^{\mathrm{N} / \mathrm{A}}$-expressing hem1 $1 \Delta$ yeast cells produced significant catalase activity (Fig. 5D). These results indicate that vacuolar LmHRG can indeed export heme from the digestive yeast vacuole. Contrastingly, hemin supplementation did not increase catalase activity in $\mathrm{LmHR} 1^{\mathrm{N} / \mathrm{A}}$-expressing hem1 $1 \Delta$ yeast (Fig. 5D), in agreement with its absence at the plasma membrane.

\section{Lysosomal LmHR1 rescue heme from endocytosed $\mathrm{Hb}$ in L. major}

Finally, we analyzed LmHR1's involvement in heme salvage from $\mathrm{Hb}$ in $L$. major. A GFP version was initially expressed in $L$. major in order to study the protein's localization. As with LaHR1 (Huynh et al., 2012), LmHR1 was present at the plasma membrane, the flagellar pocket and the endolysosomal compartment (colocalization with FM4-64 at $28^{\circ} \mathrm{C}$ ) (Fig. 6A). Intracellular LmHR1 also colocalized with endocytosed $\mathrm{Hb}$, which was added as PPIX-ApoHb, a fluorescent $\mathrm{Hb}$ derivative in which heme has been replaced by its immediate precursor PPIX (Fig. 6B). We then studied the role of LmHR1 with respect to porphyrin uptake using a non-tagged version of the transporter. In contrast with the results published with LaHR1 in L. amazonensis, in our experiments LmHR1 overexpression did not lead to a significant increase in fluorescent porphyrin uptake in L. major (Fig. 6C). To further explore the role of LmHR1 in heme uptake, we deleted one allele of $L m H R 1$ and replaced it with a hygromycin resistance gene cassette (SI Appendix Fig. S1). As described in $L$. amazonensis, we could not produce a double KO line, suggesting that the gene is also essential in $L$. major. This heterozygous knock out line $\left(L m H R 1^{+/-}\right)$accumulated $60 \%$ less fluorescent porphyrin than control cells (Fig. 6C). When an ectopic LmHR1 was expressed in $L m H R 1^{+/-}$cells the parasite's porphyrin uptake capacity was fully restored (Fig. 6C), providing supporting evidence of LmHR1's role in heme uptake. Unlike T. brucei, Leishmania has a ferrochelatase gene and can therefore insert iron into the precursor PPIX in order to synthesize heme. Thus, the bioavailability of porphyrins from endocytosed $\mathrm{Hb}$ could be measured by analyzing the de novo heme formation at the mitochondria of parasites incubated with the $\mathrm{Hb}$ derivative PPIX-ApoHb (CamposSalinas et al., 2011). Figure 6D shows that heme formation correlated with LmHR1 levels. Thus, LmHR1 overexpression increased heme formation from PPIX$\mathrm{ApoHb}$ by threefold, whereas the deletion of a $L m H R 1$ allele reduced it by $50 \%$. $L m H R 1^{+/-}$parasites complemented with LmHR1 showed similar levels of heme formation to those of the control cells. As observed in yeast, LmHR1 levels did not affect the endocytosis of $\mathrm{Hb}$ in Leishmania (data not shown).

\section{Discussion}

An Achilles' heel of trypanosomatid parasites is their absolute dependence on scavenging heme from their hosts. Proteins involved in the salvage of this porphyrin could therefore constitute new drug targets for the treatment of the neglected diseases produced by such parasites. In this paper we unravel the mechanism used by $T$. brucei and $L$. major to salvage heme from endocytosed $\mathrm{Hb}$.

Bloodstream forms of $T$. brucei, the clinically relevant form of the parasite, obtain heme exclusively through endocytosis of $\mathrm{Hb}-\mathrm{Hp}$ complexes (Vanhollebeke et al., 2008). Leishmania, on the other hand, can obtain heme by its direct import through the essential porphyrin transporter LHR1 (Huynh et al., 2012), a protein from the HRG family (Rajagopal et al., 2008), or via endocytosis of $\mathrm{Hb}$ (Sengupta et al., 1999). In both parasites, heme released after the lysosomal breakdown of $\mathrm{Hb}$ must cross the lysosome membrane before being incorporated into hemeproteins. Here, we provide evidence which strongly suggests that HRG transporters from Trypanosoma and Leishmania are responsible for this essential step. Firstly, both LmHR1 and the product of its syntenic gene in T. brucei, TbHRG, are localized in endolysosomal compartments of Leishmania and bloodstream forms of $T$. brucei, respectively, where internalized $\mathrm{Hb}$ is trafficked and digested (Sengupta et al., 1999; Vanhollebeke et al., 2008). In the case of Leishmania, the protein is also expressed in the plasma membrane, where it has been proposed to transport heme from the environment (Huynh et al., 2012). A dual localization of LaHR1 in plasma membranes and acidic compartments was also observed when the protein was expressed in HeLa cells (Huynh et al., 2012), when TbHRG and LmHR1 were expressed in yeast or in the case of human HRG1 (O'Callaghan et al., 2010). Interestingly, LmHR1 has the same exclusive intracellular localization as TbHRG when expressed in bloodstream forms of T. brucei. Secondly, both TbHRG and LmHR1 [as LaHR1 (Huynh et al., 2012)] mediate heme transport in yeast, as they rescue the growth defect of a heme auxotrophic yeast strain in the presence of low hemin concentrations. Similar rescue phenotypes of the mutant yeast strain have also been described for the HRG proteins CeHRG1, CeHRG4 and HsHRG1 (Yuan et al., 
A

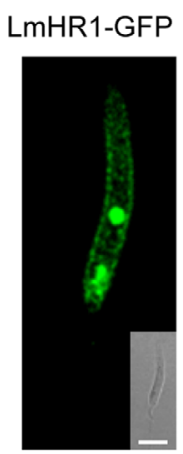

FM4-64

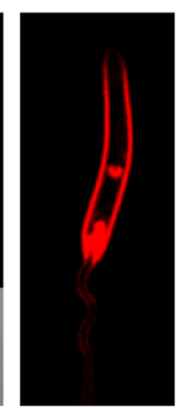

C
B

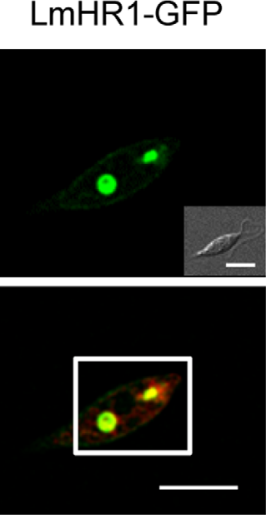

Merge
Hb-red

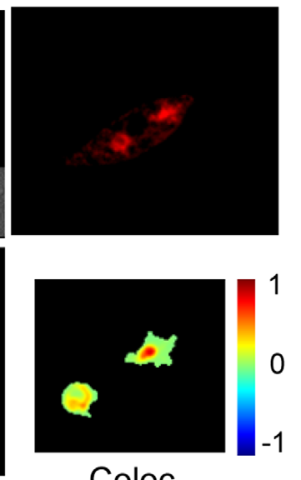

Coloc.

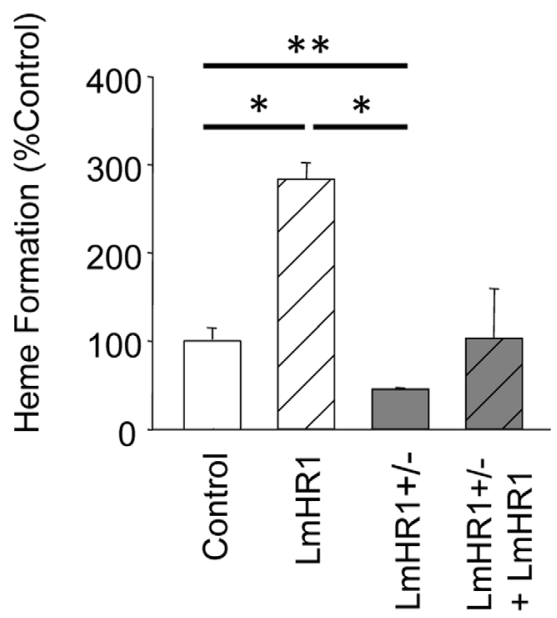

Fig. 6. LmHR1 rescues heme from endocytosed $\mathrm{Hb}$ in $L$. major promastigotes.

A. LmHR1 has dual localization at the plasma membrane and the endolysosomal compartment of Leishmania. L. major promastigotes expressing LmHR1-GFP (green) were incubated at $28^{\circ} \mathrm{C}$ with $5 \mu \mathrm{M}$ of FM4-64 (red), a probe to stain the plasma membrane and the endocytic pathway, and observed using fluorescence microscopy as indicated in Fig. 1C.

B. Intracellular LmHR1 colocalized with endocytosed Hb. L. major promastigotes expressing LmHR1-GFP (green) were incubated at $28^{\circ} \mathrm{C}$ in the presence of $10 \mu \mathrm{M}$ of the fluorescent $\mathrm{Hb}$ derivative PPIX-ApoHb (Hb-red) for 30 min and observed using fluorescence microscopy as indicated in Fig. 1C.

C. LmHR1 role in porphyrin uptake in Leishmania. The indicated strains of L. major promastigotes were incubated with $10 \mu \mathrm{M} Z \mathrm{ZMP}$ for $10 \mathrm{~min}$ and their intracellular fluorescence analyzed by flow cytometer. Control: parasites with endogenous LmHR1 levels; LmHR1: parasites overexpressing LmHR1; LmHR1 ${ }^{+/-}$: parasites with a $L m H R 1$ allele eliminated; LmHR1 $1^{+1-}+L m H R 1$ : LmHR1 ${ }^{+/-}$parasites transfected with the LmHR1 containing plasmid. The results represent the mean \pm SD of three independent experiments. $P$ values * $<0.007$.

D. LmHR1 mediates $\mathrm{Hb}$-derived heme transport from endolysosome to the cytosol in $L$. major. The mitochondrial synthesis of heme from its precursor PPIX in the form of PPIX-ApoHb was measured as described in Materials and Methods after incubating the indicated $L$. major strains with $0.125 \mu \mathrm{M}$ PPIX-ApoHb. The results represent the mean \pm SD of three independent experiments. $P$ values ${ }^{*}<0.005$ and ${ }^{*}<0.0005$.

2012). Thirdly, vacuolar TbHRG and LmHR1 are functional as they also rescue the growth of the aforementioned mutant yeast strain when hemin was substituted by $\mathrm{Hb}$, which is known to reach the vacuole after its endocytosis (Weissman et al., 2008). The capacity of these HRG proteins to use $\mathrm{Hb}$ as a source of heme in yeast was further confirmed by the fact that they promote catalase activity, which is used as a hemecontaining reporter enzyme, following yeast incubation with $\mathrm{Hb}$. This was confirmed using a LmHR1 mutant that localized exclusively to the vacuole and that also facilitated the use of heme derived from $\mathrm{Hb}$. Finally, overexpression of lysosomal TbHRG increases cytosolic heme levels from endocytosed $\mathrm{Hp}-\mathrm{Hb}$ in $\mathrm{T}$. brucei and lysosomal LmHR1 promotes the bioavailability of porphyrins bound to endocytosed $\mathrm{Hb}$ in Leishmania parasites. Furthermore, TbHRG function in T. brucei can be complemented by LmHR1 expression. Therefore, it is likely that both proteins have a similar role in endolysosomal compartments rescuing heme from endocytosed $\mathrm{Hb}$. Similarly, during erythrophagocytosis mammal HRG1 is responsible for heme transport from the phagolysosome of macrophages to the cytosol (Delaby et al., 2012; White et al., 2013), whereas CeHRG1 has been 
proposed to facilitate intracellular heme availability through endolysosomal compartments in C. elegans (Rajagopal et al., 2008).

The presented data significantly advance our understanding of the mechanisms by which heme auxotrophic trypanosomatid parasites salvage heme from their hosts (Fig. 7). Thus, bloodstream-form T. brucei endocytoses $\mathrm{Hp}-\mathrm{Hb}$ complexes through the $\mathrm{Hb}-\mathrm{Hp}$ receptor (Vanhollebeke et al., 2008). $\mathrm{Hb}$ is then trafficked through the endocytic pathway, digested in the endolysosomal compartment and the free heme released is transported by TbHRG to the cytosol (this work), where it is incorporated in hemeproteins such as sterol $14 \alpha$-demethylase (Lepesheva et al., 2010). Leishmania can internalize heme through plasma membrane LHR1 (Huynh et al., 2012) or it can also use heme bound to $\mathrm{Hb}$ (Chang and Chang, 1985; Campos-Salinas et al., 2011). Hb bound to its receptor, a hexokinase, is endocytosed (Sengupta et al., 1999; Krishnamurthy et al., 2005) and routed to the late endosomes/lysosomes where it is degraded and releases the heme moiety (Singh et al., 2003; Patel et al., 2008). Intracellular LHR1, located at the endolysosomal compartment, then export free heme to the cytosol (this work). The ABC transporter LABCG5 probably also plays a role at this point, as it has been shown to be involved in the traffic of $\mathrm{Hb}$-derived heme to the mitochondria (Campos-Salinas et al., 2011). LABCG5 is located in a different vesicular network unrelated to the endocytic route and, therefore, it does not promote heme export from the lysosomes (Campos-Salinas et al., 2011). However, at its location, LABCG5 could take up the heme exported by LHR1 from the endo-lysosomes where $\mathrm{Hb}$ is digested (Campos-Salinas et al., 2011), although this possibility still requires investigation. Finally, the mitochondrial $A B C$ transporter $\angle A B C B 3$, which is involved in the maturation of cytosolic iron/sulfur clusters, also promotes mitochondrial heme biogenesis from host precursors (Martinez-Garcia et al., 2016).

The absence of HRG proteins at the plasma membrane of bloodstream $T$. brucei could be due to the low availability of free heme in the human serum, compared to $\mathrm{Hp}-\mathrm{Hb}$ levels (Thomsen et al., 2013). In contrast, TbHRG (and LHR1) localizes to the plasma membrane and endolysosomes in procyclic $T$. brucei parasites (M. Cabello-Donayre and J.M. Pérez-Victoria, unpublished results), suggesting that this stage of the parasite could also take up free heme released after blood digestion inside the tsetse fly. Likewise, the double localization of HRG proteins at the plasma membrane and intracellular compartments observed in Leishmania amastigotes and promastigotes (Huynh et al., 2012) could be also related to the exposition to both, free porphyrins and $\mathrm{Hb}$, in the macrophage phagolysosome and inside the insect vector.

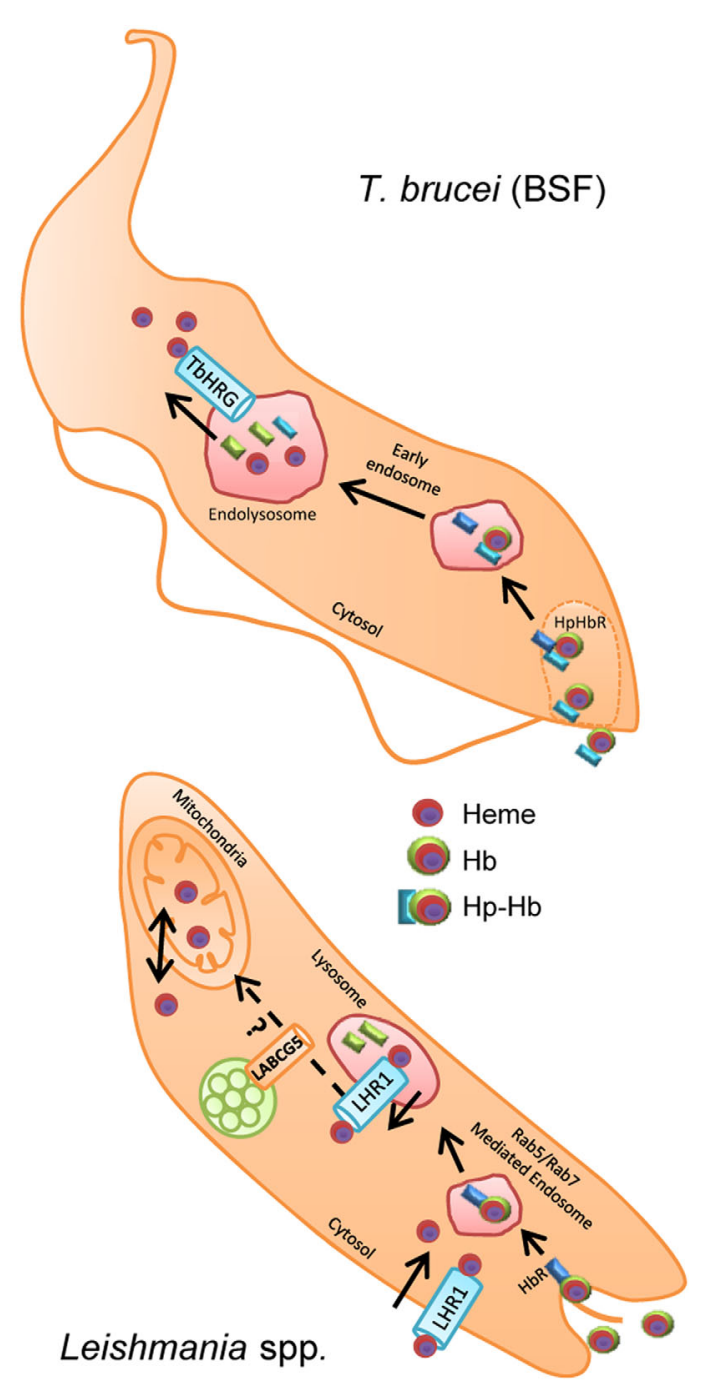

Fig. 7. Proposed models for the role of HRG transporters in bloodstream T. brucei and Leishmania spp. Bloodstream forms of T. brucei endocytose $\mathrm{Hp}-\mathrm{Hb}$ complexes through the $\mathrm{Hp}-\mathrm{Hb}$ receptor $(\mathrm{HpHbR}) . \mathrm{Hb}$ is then trafficked through the endocytic route and digested in the endolysosomal compartment releasing the heme moiety. Heme is subsequently exported to the cytosol through TbHRG. In Leishmania promastigotes, heme could enter the cells through plasma membrane LHR1. In addition, $\mathrm{Hb}$ is internalized by receptor $(\mathrm{HbR})$ mediated endocytosis and trafficked through the endocytic pathway to the lysosome, where $\mathrm{Hb}$ is degraded and heme released. This heme is exported from the lysosome by intracellular LHR1 and reaches the mitochondria with the participation of the ABC transporter LABCG5, which is located in a vesicular network unrelated to the endocytic route.

We have also shown that TbHRG is essential for the clinically relevant bloodstream forms of $T$. brucei. The only source of heme in this stage of the parasite's lifecycle is considered to be the receptor $(\mathrm{HpHbR})$-mediated endocytosis of $\mathrm{Hp}-\mathrm{Hb}$ complexes (Vanhollebeke et al., 2008), a process well characterized because of its relation with humans' natural immunity against many trypanosome species (Vanhollebeke et al., 2008; Vanhollebeke and Pays, 2010; Pays et al., 2014). The abrogation of TbHRG 
expression hampers this means of salvaging heme from the infected host and can explain why it is essential. However, when knocking out the $\mathrm{TbHpHbR}$ gene, the growth of the trypanosomes has been shown to be severely compromised (Vanhollebeke et al., 2008) or slightly affected (DeJesus et al., 2013), in either case suggesting that heme scavenging through $\mathrm{HpHbR}$ may not be essential for the parasite (DeJesus et al., 2013). It has therefore been suggested that $T$. brucei $\mathrm{HpHbR}^{-1-}$ parasites employ other heme uptake mechanisms which compensate for the loss of the $\mathrm{Hp}-\mathrm{Hb}$ receptor (DeJesus et al., 2013). This possibility remains to be proven, but the essential nature of TbHRG suggests that this putative alternative mechanism for heme scavenging also relies on the traffic of any form of heme to the parasite's endolysosome, requiring TbHRG for heme export to the cytoplasm. LHR1 is also essential for L. amazonensis (Huynh et al., 2012) (and probably for L. major) and required for the parasite's virulence in animal models (Miguel et al., 2013; Renberg et al., 2015). Although we have not analyzed the role of the syntenic HRG gene of T. cruzi, an essential role could also be expected for TcHRG, as T. cruzi, responsible for Chagas disease, is also auxotrophic for heme and performs $\mathrm{Hb}$ endocytosis (Lara et al., 2007). The low similarity between trypanosomatid HRG proteins and the unique human HRG protein (14-18\% identity) could enable the development of specific inhibitors against parasite transporters. Therefore, the specific targeting of trypanosomatid HRG proteins represents a promising route for controlling the neglected diseases produced by the aforementioned parasites.

In conclusion, we show that heme auxotrophic trypanosomatid parasites rescue heme from endocytosed $\mathrm{Hb}$ through endolysosomal HRG transporters. As these proteins are essential and present a low degree of similarity with their human orthologue, their pharmacological targeting could constitute a novel method to fight these parasites.

\section{Experimental procedures}

\section{Chemical compounds}

DAPI dihydrochloride, MES hydrate, yeast synthetic drop-out medium supplements without uracil, lithium acetate dihydrate, Triton X-100 (4-(1,1,3,3-tetramethylbutyl)phenyl-polyethylene glycol), $\mathrm{Hb}$ and hemin were all obtained from Sigma. PPIX-Na and ZnMPIX were from Frontier Scientific. Dextran Alexa 647 (10,000 MW) and FM4-64 were supplied by Molecular Probes (Invitrogen). Agar was from Intron Biotechnology.

\section{Strains, culture conditions DNA constructs and cell transfection}

'Single marker' T. brucei Lister 427 bloodstream cell line S16 (Wirtz et al., 1999) was cultured HMI-9 medium containing
$10 \%$ heat inactivated fetal bovine serum (hiFBS) as described in (Carvalho et al., 2015). T. brucei 449 procyclic cells (Biebinger et al., 1997) were grown in SDM-79 medium containing $10 \%$ hiFBS and $7.5 \mu \mathrm{g} \mathrm{ml}^{-1}$ hemin. Cells were transfected and selected for the corresponding resistance drugs as described in (Clayton et al., 2005). TbHRG from T. brucei (Tb927.8.6010) was isolated from genomic DNA of $T$. brucei by PCR using primers pairs $\mathrm{P} 6$ and $\mathrm{P} 7$ (see all primers used in Table S2), cloned in fusion with GFP (C-terminal) into the expression vector pLEW100 (Wirtz et al., 1999). RNAi studies were performed as described in (Clayton et al., 2005). Briefly, a fragment of TbHRG was PCR-amplified (primer pairs P1 and P2) and cloned into p2T7Blue to yield p2T7-TbHRGi. Bloodstream trypanosomes were transfected with linearized p2T7TbHRGi and selected in the presence of $5 \mu \mathrm{g} \mathrm{ml}^{-1}$ hygromycin. RNAi was induced with $1 \mu \mathrm{g} \mathrm{ml}^{-1}$ doxycycline. Trypanosomes containing P2T7-TbHRGi were also transfected with the inducible expression plasmid pLEW100 containing LmHR1 (LmjF24.2230) fused to GFP (C-terminal). LmHR1 was isolated from genomic DNA of $L$. major by PCR using primers pairs P11 and P12. Promastigote-form L. major (MHOM/IL/80/ Friedlin) was cultured in RPMI 1640 modified medium supplemented with $10 \%$ hiFBS as described in (Arana et al., 1998). LmHR1 was isolated by PCR (primers pairs P13 and P14) and cloned into the Leishmania expression vectors pXG (Strain B1288) and pXG-/GFP+ (strain B2863) (Ha et al., 1996). Promastigotes were transfected with the different constructs and selected for the corresponding resistance drugs as described previously (Parodi-Talice et al., 2003). S. cerevisiae BY4742

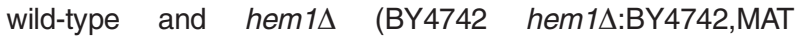
$\alpha$;his3 $\Delta 1$;leu2 $\Delta 0$;lys2 $\Delta 8$; ura3 $\Delta 0$; hem1::LEU2) were grown at $30^{\circ} \mathrm{C}$ in YPD (yeast extract-peptone-dextrose) or appropriate synthetic complete (SC) medium supplemented with $250 \mu \mathrm{M}$ $\delta$-aminolevulinic acid hydrochloride (ALA), hemin or $\mathrm{Hb}$. TbHRG and LmHR1 were amplified by PCR using primers pairs P3/P4, P3/P5, P8/P9 and P8/P10 before being cloned into the yeast expression vectors pYESDEST52 and pDR (GFP fusion). Yeast was transformed using the lithium acetate medium (Ito et al., 1983) and transformants were selected on $2 \% \mathrm{w} / \mathrm{v}$ glucose SC (-Ura) plates.

\section{LmHR1 gene deletion}

Targeted gene replacement of the L. major LmHR1 gene was performed as we have previously described (MartinezGarcia et al., 2016) for the case of the L. major LmABCB3 gene. Briefly, a targeting DNA fragment was constructed in which the hyg gene (flanked by untranslated regions of the L. major DHFR-TS gene (248 bp of the 5'-UTR and $869 \mathrm{bp}$ of the $\left.3^{\prime}-U T R\right)$, conferring resistance to hygromycin $B$, was flanked by LmHR1 upstream (primers pairs P15 and P16) and downstream (P17 and P18) regions (Fig. S1). The different fragments were amplified by PCR from genomic DNA using the indicated primers, subcloned into pGEM-T vector (Promega), and then assembled in this vector. Log phase $L$. major promastigotes were transfected with $5 \mu \mathrm{g}$ of the linearized DNA targeting constructions, generated by Apal and Notl digestion, through use of the Amaxa Nucleofector System (Lonza). Transfected parasites were selected 
with $50 \mu \mathrm{g} \mathrm{ml}^{-1}$ hygromycin B and cloned in semi-solid culture medium ( $1 \%$ agar).

\section{Fluorescence microscopy}

Bloodstream-form T. brucei expressing TbHRG-GFP or LmHR1-GFP was incubated with $2.5 \mathrm{mg} \mathrm{ml}^{-1}$ of Dextran Alexa 647 for $30 \mathrm{~min}$ at $37^{\circ} \mathrm{C}$ as described (Engstler et al., 2005). $L$. major promastigotes expressing LmHR1-GFP were incubated with $10 \mu \mathrm{M}$ PPIX-ApoHb for $60 \mathrm{~min}$ at $28^{\circ} \mathrm{C}$ in HPMI medium [T. brucei cannot internalize this free compound as the receptor $\mathrm{TbHpHbR}$ only recognizes the complex $\mathrm{Hp}-\mathrm{Hb}$ (Vanhollebeke et al., 2008)]. Hem1s yeast expressing TbHRG-GFP or LmHR1-GFP (Wt or mutated) were used in the exponential growth phase. Cells were washed twice in $500 \mu \mathrm{l}$ of cold phosphate-buffered saline (PBS), fixed and processed by microscope observation. Images were acquired using Olympus epifluorescence and Leica SP5 confocal microscopes, while the stacks $(n=40)$ were deconvolved using Huygens Professional image processing software from Scientific Volume Imaging (http://www.svi.nl). Statistical colocalization analyses were performed on the 3D- sections with Colocalization Colormap software from FIJI (http://www.fiji.sc/Fiji). The index of correlation (Icorr) represents the fraction of positively correlated pixels in the 3D-images. The results show a representative Z- projection image.

\section{Gene expression analysis}

Total RNA from the indicated Trypanosoma strains was prepared using the total RNA isolation kit (Roche Biochemicals). cDNA was synthesized from $1 \mu \mathrm{g}$ of total RNA using qSCRIPT ${ }^{\mathrm{TM}}$ CDNA Synthesis kit (Quanta Biosciences Inc.) according to the manufacturer's instructions. The cDNA obtained was amplified with primers pairs P19 and P20 for TbHRG and primers pairs P21 and P22 for LmHR1. Actin (primers pairs P23 and P24) and tubulin (primers pairs P25 and P26) were used as housekeeping genes. Quantitative PCR was performed with iTaq Universal SYBR Green ${ }^{\circledR}$ Supermix (BIORAD). Relative transcript abundance of TbHRG was calculated using CFX Manager Software, with $\Delta \Delta \mathrm{Cq}$ method (Livak and Schmittgen, 2001), using as control the TbHRG expression in bloodstream forms.

\section{Yeast growth assays}

The spot growth assay was performed as described in (Yuan et al., 2012) but also including the indicated $\mathrm{Hb}$ concentration on the plates. Control, TbHRG- and LmHR1expressing hem1s yeast were spotted in each plate. The liquid assay was performed similarly, but instead of spot plates heme-depleted cells were suspended in a medium to an $\mathrm{OD}_{600}$ of 0.1 and grown with the indicated concentrations of hemin or $\mathrm{Hb} . \mathrm{OD}_{600}$ was measured after $24 \mathrm{~h}$ incubation at $30^{\circ} \mathrm{C}$ and $180 \mathrm{rpm}$.

\section{Catalase reporter assay}

Catalase activity was measured as described in (Iwase et al., 2013) but with some modifications. Briefly, yeast cells were cultured for $24 \mathrm{~h}$ without ALA to deplete heme, and then incubated for $16 \mathrm{~h}$ in the absence or presence of either $0.5 \mu \mathrm{M} \mathrm{Hb}$ or $2 \mu \mathrm{M}$ hemin. Washed yeast cells were then adjusted to a $\mathrm{OD}_{600}$ of $5 \mathrm{in} 1 \mathrm{ml}$ of $\mathrm{H}_{2} \mathrm{O} .300 \mu \mathrm{l}$ of $1 \%$ Triton X-100 and $300 \mu \mathrm{l}$ of $30 \%$ hydrogen peroxide (Sigma) were then added. Catalase-generated oxygen bubbles were trapped by Triton X100 and observed as a foam, the height of which was proportional to the enzyme activity.

\section{Cytosolic heme levels in bloodstream-form T. brucei}

Cytosolic heme levels in bloodstream-form T. brucei were measured after gently lysis of the parasites as described in (da Silva Augusto et al., 2015). Briefly, parasites (grown in HMI-9 supplemented with $10 \%$ hiFBS as a source of $\mathrm{Hp}-\mathrm{Hb}$ ) were washed and lysed by freezing and thawing (three cycles). The insoluble material was removed by centrifugation at $14,000 \mathrm{~g}$ for $10 \mathrm{~min}$ and cytosolic heme were measured with the Hemin Assay Kit (Sigma) and normalized for the amount of protein as described in (MartinezGarcia et al., 2016).

\section{Uptake of ZnMP in Leishmania}

Porphyrin uptake in Leishmania promastigotes was performed as previously described (Campos-Salinas et al., 2011) but with some modifications. Briefly, washed parasites were suspended in HPMI medium (20 mM HEPES pH 7.25, $132 \mathrm{mM} \mathrm{NaCl}, 3.5 \mathrm{mM} \mathrm{KCl}, 0.5 \mathrm{mM} \mathrm{MgCl}, 1 \mathrm{mM}$ $\mathrm{CaCl}_{2}, 5 \mathrm{mM}$ glucose) and incubated with $10 \mu \mathrm{M}$ ZnMP at $28^{\circ} \mathrm{C}$ for $10 \mathrm{~min}$. Parasites were then washed with ice-cold PBS containing $5 \%$ BSA and fixed with $2 \%$ paraformaldehyde in PBS. Intracellular fluorescence was measured using flow cytometry (excitation at $405 \mathrm{~nm}$ and emission at between 575 and $585 \mathrm{~nm}$ ) employing a FacsAria Cell Sorter III (Becton Dickinson, USA).

\section{Analysis of de novo synthesized heme in Leishmania}

ApoHb-PPIX was prepared as described in (Campos-Salinas et al., 2011). Heme-depleted serum was prepared as described in (Carvalho et al., 2009) after filtration of hiFBS over Amicon Ultra 10,000 MWCO columns (Millipore, Carrigtwohill, Co. Cork, Ireland) to select the low molecularweight fraction of FBS. Heme depletion was verified by measuring the optical absorbance at $405 \mathrm{~nm}$ (Huynh et al., 2012). This heme-depleted serum allows normal Leishmania growth only when a heme source (hemin or $\mathrm{Hb}$ ) is provided (data not shown). Synthesis of heme from its precursor $\mathrm{PPIX}$, in the form of PPIX-ApoHb, in Leishmania was performed as previously described (Campos-Salinas et al., 2011) but with some modifications. Briefly, parasites were incubated in culture medium supplemented with $10 \%$ hemedepleted serum for $16 \mathrm{~h}$ with or without $0.125 \mu \mathrm{M}$ PPIX$\mathrm{ApoHb}$ at $28^{\circ} \mathrm{C}$. Parasites were then washed and lysed by six freeze/thaw cycles with liquid nitrogen before intracellular heme levels were measured using the Hemin Assay Kit (Sigma) and normalized for the amount of protein as above described. De novo synthesized heme was the difference 
between heme levels measured in parasites incubated in the presence and absence of PPIX-ApoHb.

\section{Statistical analysis}

Experiments were performed three times in duplicate. All data are presented as the mean and the error represents the S.D. Statistical significance was determined using Student's t-test. Significance was considered to be $P<0.05$.

\section{Note added in proof}

While our manuscript was under revision, Merli and coworkers reported the characterization of TcHTE, the T. cruzi ortholog of TbHRG [Merli et al., 2016]. The authors showed that TcHTE is located in the flagellar pocket of the parasites and postulate TcHTE as a protein involved in improving the efficiency of the heme uptake or trafficking in T. cruzi.

\section{Acknowledgements}

We thank Ivan Hapala (IABG-SAS, Slovakia), Stephen M. Beverley (Washington University School of Medicine, USA) and Olivier Cagnac (EEZ-CSIC, Spain) for kindly providing, respectively, the hem $1 \Delta$ yeast strain, the Leishmania and the yeast vectors used throughout this research work. We thank Ignacio Pérez-Victoria for the preparation of $\mathrm{ApoHb}$. We are grateful to Jean Mathieu Bart (IPBLN-CSIC, Spain) for helpful discussions. This work was supported by Spanish grants BIO1786 (JMPV) from the Junta de Andalucía and SAF2011-28215 (JMPV) and BFU2014-55193-P (AE) from the Ministerio de Economía y Competitividad and by FEDER funds from the EU to JMPV and AE. MCD was a student of the $\mathrm{PhD}$ program "Biochemistry and Molecular Biology" of the University of Granada (Spain). MCD was recipient of a FPU fellowship from the Spanish Ministerio de Educación, Cultura y Deporte; SMC was recipient of a JAEDOC from the Spanish CSIC (Ministerio de Economía y Competitividad), cofounded by the Fondo Social Europeo, LMOZ was recipient of a Colciencias fellowship from the Colombian Ministerio de Ciencia, Tecnología e Innovación; MMG was recipient of a FPI fellowship from the Spanish Ministerio de Economía y Competitividad. The authors declare that they have no conflict of interest.

\section{References}

Alsford, S., Turner, D.J., Obado, S.O., Sanchez-Flores, A., Glover, L., Berriman, M., et al. (2011). High-throughput phenotyping using parallel sequencing of RNA interference targets in the African trypanosome. Genome Res 21: 915-924.

Arana, F.E., Perez-Victoria, J.M., Repetto, Y., Morello, A., Castanys, S., and Gamarro, F. (1998). Involvement of thiol metabolism in resistance to glucantime in Leishmania tropica. Biochem Pharmacol 56: 1201-1208.

Biebinger, S., Wirtz, L.E., Lorenz, P., and Clayton, C. (1997). Vectors for inducible expression of toxic gene products in bloodstream and procyclic Trypanosoma brucei. Mol Biochem Parasitol 85: 99-112.

Campos-Salinas, J., Cabello-Donayre, M., GarciaHernandez, R., Perez-Victoria, I., Castanys, S., Gamarro, F., and Perez-Victoria, J.M. (2011). A new ATP-binding cassette protein is involved in intracellular haem trafficking in Leishmania. Mol Microbiol 79: 1430-1444.

Carvalho, L., Martinez-Garcia, M., Perez-Victoria, I., Manzano, J.I., Yardley, V., Gamarro, F., and PerezVictoria, J.M. (2015). The oral antimalarial drug tafenoquine shows activity against Trypanosoma brucei. Antimicrob Agents Chemother 59: 6151-6160.

Carvalho, S., Cruz, T., Santarem, N., Castro, H., Costa, V., and Tomas, A.M. (2009). Heme as a source of iron to Leishmania infantum amastigotes. Acta Trop 109: 131-135.

Chang, C.S., and Chang, K.P. (1985). Heme requirement and acquisition by extracellular and intracellular stages of Leishmania mexicana amazonensis. Mol Biochem Parasitol 16: 267-276.

Clayton, C.E., Estevez, A.M., Hartmann, C., Alibu, V.P., Field, M., and Horn, D. (2005). Down-regulating gene expression by RNA interference in Trypanosoma brucei. Methods Mol Biol 309: 39-60.

Cupello, M.P., Souza, C.F., Buchensky, C., Soares, J.B., Laranja, G.A., Coelho, M.G., et al. (2011). The heme uptake process in Trypanosoma cruzi epimastigotes is inhibited by heme analogues and by inhibitors of $A B C$ transporters. Acta Trop 120: 211-218.

Da Silva Augusto, L., Moretti, N.S., Ramos, T.C., De Jesus, T.C., Zhang, M., Castilho, B.A., and Schenkman, S. (2015). A membrane-bound elF2 alpha kinase located in endosomes is regulated by heme and controls differentiation and ROS levels in Trypanosoma cruzi. PLoS Pathog 11: e1004618.

Dejesus, E., Kieft, R., Albright, B., Stephens, N.A., and Hajduk, S.L. (2013). A single amino acid substitution in the group 1 Trypanosoma brucei gambiense haptoglobinhemoglobin receptor abolishes TLF-1 binding. PLOS Pathog 9: e1003317.

Delaby, C., Rondeau, C., Pouzet, C., Willemetz, A., Pilard, N., Desjardins, M., and Canonne-Hergaux, F. (2012). Subcellular localization of iron and heme metabolism related proteins at early stages of erythrophagocytosis. PLoS One 7: e42199.

Engstler, M., Weise, F., Bopp, K., Grunfelder, C.G., Gunzel, M., Heddergott, N., and Overath, P. (2005). The membrane-bound histidine acid phosphatase TbMBAP1 is essential for endocytosis and membrane recycling in Trypanosoma brucei. J Cell Sci 118: 2105-2118.

Guha, R., Gupta, D., Rastogi, R., Vikram, R., Krishnamurthy, G., Bimal, S., et al. (2013). Vaccination with leishmania hemoglobin receptor-encoding DNA protects against visceral leishmaniasis. Sci Transl Med 5: 202ra121.

Ha, D.S., Schwarz, J.K., Turco, S.J., and Beverley, S.M. (1996). Use of the green fluorescent protein as a marker in transfected Leishmania. Mol Biochem Parasitol 77: 57-64.

Huynh, C., Yuan, X., Miguel, D.C., Hamza, I., and Andrews, N.W. (2012). Heme uptake in Leishmania amazonensis is 
mediated by the transmembrane protein LHR1. PLoS Pathog 8: e1002795.

Ito, H., Fukuda, Y., Murata, K., and Kimura, A. (1983). Transformation of intact yeast cells treated with alkali cations. J Bacteriol 153: 163-168.

Iwase, T., Tajima, A., Sugimoto, S., Okuda, K., Hironaka, I., Kamata, Y., et al. (2013). A simple assay for measuring catalase activity: A visual approach. Sci Rep 3: 3081.

Kathiresan, M., Martins, D., and English, A.M. (2014). Respiration triggers heme transfer from cytochrome c peroxidase to catalase in yeast mitochondria. Proc Natl Acad Sci USA 111: 17468-17473.

Kohut, P., Wustner, D., Hronska, L., Kuchler, K., Hapala, I., and Valachovic, M. (2011). The role of ABC proteins Aus1p and Pdr11p in the uptake of external sterols in yeast: Dehydroergosterol fluorescence study. Biochem Biophys Res Commun 404: 233-238.

Krishnamurthy, G., Vikram, R., Singh, S.B., Patel, N., Agarwal, S., Mukhopadhyay, G., et al. (2005). Hemoglobin receptor in Leishmania is a hexokinase located in the flagellar pocket. J Biol Chem 280: 5884-5891.

Lara, F.A., Sant'anna, C., Lemos, D., Laranja, G.A., Coelho, M.G., Reis Salles, I., et al. (2007). Heme requirement and intracellular trafficking in Trypanosoma cruzi epimastigotes. Biochem Biophys Res Commun 355: 16-22.

Lepesheva, G.I., Park, H.W., Hargrove, T.Y., Vanhollebeke, B., Wawrzak, Z., Harp, J.M., et al. (2010). Crystal structures of Trypanosoma brucei sterol 14alpha-demethylase and implications for selective treatment of human infections. J Biol Chem 285: 1773-1780.

Livak, K.J., and Schmittgen, T.D. (2001). Analysis of relative gene expression data using real-time quantitative PCR and the 2(-Delta Delta C(T)) Method. Methods 25: 402-408.

Lwoff, M. (1933). Remarques sur la nutrition des Trypanosomides et des bactéries parahémotrophes le "fer actif" de baudisch. Ann Inst Pasteur 51: 707-713.

Martinez-Garcia, M., Campos-Salinas, J., Cabello-Donayre, M., Pineda-Molina, E., Galvez, F.J., Orrego, L.M., et al. (2016). LmABCB3, an atypical mitochondrial ABC transporter essential for Leishmania major virulence, acts in heme and cytosolic iron/sulfur clusters biogenesis. Parasit Vectors 9: 7.

Miguel, D.C., Flannery, A.R., Mittra, B., and Andrews, N.W. (2013). Heme uptake mediated by LHR1 is essential for Leishmania amazonensis virulence. Infect Immun 81: 3620-3626.

O'callaghan, K.M., Ayllon, V., O'keeffe, J., Wang, Y., Cox, O.T., Loughran, G., et al. (2010). Heme-binding protein HRG-1 is induced by insulin-like growth factor I and associates with the vacuolar $\mathrm{H}+$-ATPase to control endosomal $\mathrm{pH}$ and receptor trafficking. $J$ Biol Chem 285: 381-391.

Parodi-Talice, A., Araujo, J.M., Torres, C., Perez-Victoria, J.M., Gamarro, F., and Castanys, S. (2003). The overexpression of a new $A B C$ transporter in Leishmania is related to phospholipid trafficking and reduced infectivity. Biochim Biophys Acta 1612: 195-207.

Patel, N., Singh, S.B., Basu, S.K., and Mukhopadhyay, A. (2008). Leishmania requires Rab7-mediated degradation of endocytosed hemoglobin for their growth. Proc Natl Acad Sci USA 105: 3980-3985.
Pays, E., Vanhollebeke, B., Uzureau, P., Lecordier, L., and Perez-Morga, D. (2014). The molecular arms race between African trypanosomes and humans. Nat Rev Microbiol 12: 575-584.

Rajagopal, A., Rao, A.U., Amigo, J., Tian, M., Upadhyay, S.K., Hall, C., et al. (2008). Haem homeostasis is regulated by the conserved and concerted functions of HRG1 proteins. Nature 453: 1127-1131.

Renberg, R.L., Yuan, X., Samuel, T.K., Miguel, D.C., Hamza, I., Andrews, N.W., and Flannery, A.R. (2015). The heme transport capacity of LHR1 determines the extent of virulence in Leishmania amazonensis. PLoS Negl Trop Dis 9: e0003804.

Sengupta, S., Tripathi, J., Tandon, R., Raje, M., Roy, R.P., Basu, S.K., and Mukhopadhyay, A. (1999). Hemoglobin endocytosis in Leishmania is mediated through a $46-\mathrm{kDa}$ protein located in the flagellar pocket. $J$ Biol Chem 274: 2758-2765.

Singh, S.B., Tandon, R., Krishnamurthy, G., Vikram, R., Sharma, N., Basu, S.K., and Mukhopadhyay, A. (2003). Rab5-mediated endosome-endosome fusion regulates hemoglobin endocytosis in Leishmania donovani. Embo $J$ 22: 5712-5722.

Stodkilde, K., Torvund-Jensen, M., Moestrup, S.K., and Andersen, C.B. (2014). Structural basis for trypanosomal haem acquisition and susceptibility to the host innate immune system. Nat Commun 5: 5487.

Thomsen, J.H., Etzerodt, A., Svendsen, P., and Moestrup, S.K. (2013). The haptoglobin-CD163-heme oxygenase-1 pathway for hemoglobin scavenging. Oxid Med Cell Longev 2013: 523652.

Vanhollebeke, B., and Pays, E. (2010). The trypanolytic factor of human serum: Many ways to enter the parasite, a single way to kill. Mol Microbiol 76: 806-814.

Vanhollebeke, B., De Muylder, G., Nielsen, M.J., Pays, A., Tebabi, P., Dieu, M., et al. (2008). A haptoglobinhemoglobin receptor conveys innate immunity to Trypanosoma brucei in humans. Science 320: 677-681.

Weissman, Z., Shemer, R., Conibear, E., and Kornitzer, D. (2008). An endocytic mechanism for haemoglobin-iron acquisition in Candida albicans. Mol Microbiol 69: 201-217.

White, C., Yuan, X., Schmidt, P.J., Bresciani, E., Samuel, T.K., Campagna, D., et al. (2013). HRG1 is essential for heme transport from the phagolysosome of macrophages during erythrophagocytosis. Cell Metab 17: 261-270.

Widener, J., Nielsen, M.J., Shiflett, A., Moestrup, S.K., and Hajduk, S. (2007). Hemoglobin is a co-factor of human trypanosome lytic factor. PLoS Pathog 3: 1250-1261.

Wirtz, E., Leal, S., Ochatt, C., and Cross, G.A. (1999). A tightly regulated inducible expression system for conditional gene knock-outs and dominant-negative genetics in Trypanosoma brucei. Mol Biochem Parasitol 99: 89-101.

Yuan, X., Protchenko, O., Philpott, C.C., and Hamza, I. (2012). Topologically conserved residues direct heme transport in HRG-1-related proteins. J Biol Chem 287, 4914-4924.

\section{Supporting information}

Additional supporting information may be found in the online version of this article at the publisher's web-site. 